\title{
Emergency Logistics Center Location Selection Based on Hybrid Multi-attribute
}

\author{
Jiaxin Wang ${ }^{1, a}$, Xiaoxia Zhu ${ }^{2, b}$ and Jia Jiang ${ }^{3, c}$ \\ ${ }^{1}$ Department of Basic Education, Beijing Polytechnic , Beijing, China \\ 2 School of Sciences, Hebei University of Science and Technology, Shijiazhuang, Hebei, China \\ 3 School of Economics and Management, Hebei University of Science and Technology, \\ Shijiazhuang Hebei, China \\ a wjx12231200@126.com, b zhuxiaoxia66@126.com, cjiang_jia67@163.com
}

\begin{abstract}
Keywords: Emergency Logistics Center, Hybrid Multi-attribute, Location Decision, Triangular Fuzzy Number, Synthetic Effect Funct

Abstract. In this paper, we take a full consideration to the location impact factors of emergency logistics center, which is technology, economy, environment and society and so on, based on sorting of the research results on the emergency logistics center location. Then, we build the hybrid multiple attribute index system of emergency logistics center location selection, and put forward the calculation method of a hybrid multiple attribute value transformation, fuzzy comprehensive quantitative and established the location selection model of the emergency logistics center based on Hybrid Multi-attribute decision method. Finally, we specify the model by an example. The results indicate that the method is effective, probability, and innovative.
\end{abstract}

\section{Introduction}

Major disasters that have a regional, national or even global effect happen from time to time in this world, like the 9.11 terrorist attack in the USA in 2001, the global break-out of SARS in 2003, the Indian tsunami in 2004, the Blue algae blooming in Taihu Lake in 2007, the Wenchuan earthquake on May 12, 2208, the H1N1 epidemic in 2009 and the Tsunami on March 11, 2011 as well as the disastrous nuclear leakage. All of these disasters not only cause tremendous causalities and property losses but also threaten the human survival and social development. Therefore, when dealing with various disasters, the region or the country should make systematic, scientific and reasonable emergency logistics plan, especially a better arrangement for the locations of the emergency logistics centers. Only in this way, can the logistics centers play a good role in collecting, transporting, storing, and assigning the needed materials. Meanwhile, the region's or country's ability to supply the materials in places and in time can be enhanced, too. The negative effect and damage of the disasters can be reduced. Thus, the research on a better arrangement for the locations of emergency logistics centers is not only theoretically meaningful but also of practical values.

Research on the location of the emergency facilities is very important in the field of designing locations of facilities ${ }^{[1]}$. Nowadays, there are two ways to choose the location of the facilities. One is the operational research, building a model, like the approaches of barycenter ${ }^{[2]}, \mathrm{p}$-center $^{[3]}, \mathrm{p}$-middle value modal ${ }^{[4]}$ and coverage model ${ }^{[5]}$ as well as other forms ${ }^{[6]}$. However, these models only take into consideration of cost, time separately or are improved by considering both cost and time. The other thinks about thoroughly the proper factors when designing the locations of the facilities, that is the multiple criteria decision making based on qualitative research, like the approaches of $\mathrm{AHP}^{[7]}$, fuzzy comprehensive evaluation ${ }^{[8]} \mathrm{DEA}^{[9]}$, TOPSIS ${ }^{[10]}$, and RS- $\mathrm{AHP}^{[11]}$. However, many factors will influence the logistics location, including economy, environment, technology, social elements and so on. By analyzing the features of the emergency facilities, people build a multi-attribute evaluation index, and evaluate the different plans through the multiple criteria decision-making method. Finally, the best location is chosen. Because of such, the qualitative research is a comprehensive and systematic decision-making way, and it also takes many subjective elements into consideration, such as the 
decision maker's experience, preference as well as his or her personal willingness. Nevertheless, since there are many subjective factors in the qualitative research, there will be errors in the evaluation process.

Based on what is mentioned above, after considering both the subjective and objective factors, and analyzing the approaches of designing locations for emergency logistics centers, the essay builds a location evaluation system for the emergency logistics centers based on the hybrid multi-attribute decision-making, and erases the errors of the subjective evaluation. It mainly focuses on the following work. 1).Build a hybrid multi-attribute index system for designing the locations of the emergency locations centers, an approach of hybrid multi-attribute triangular fuzzy number switch, as well as a formula to standardize the triangular fuzzy number index; 2).Starting from the features of the fuzzy information structures, it brings up a function of level effect to describe the fuzzy information dealing consciousness and builds a function of synthetic effect based on the centralized quantization value and unequal degrees; 3). It designs the hybrid multi-attribute location design method for the emergency logistics centers. Finally, the essay analyzes the characteristics of the chosen model together with the specific cases. Such work not only lays a foundation for designing the best project and performance appraisal but also enriches the theory on information dealing and comprehensive evaluation. It not only realizes easily the switch between the qualitative description and planning but also blends effectively the decision-making awareness with the process. Therefore, this essay is of great instructive value on solving the decision-making problems in uncertain circum- stances.

\section{Preliminaries}

The fuzziness is a common phenomenon in real world.In1965, Zadeh proposed the concept of the fuzzy set and established fuzzy set theory ${ }^{[12,13]}$, which provide a new effective too for the description and processing of uncertain information, and these made many theoretical researches and the application results in the field of fuzzy control, artificial intelligence, expert systems .To establish a general quantification of fuzzy information, we introduce the following definition ${ }^{[14]}$.

Definition 1 We call $L(\lambda):[0,1] \rightarrow[a, b] \subset[0, \infty)$ a level effect function, if $L(\lambda)$ is piecewise continuous and monotone non-decreasing. For $A \in E^{1}$, let

$$
I_{L}(A)=\frac{1}{2 L^{*}} \int_{0}^{1} L(\lambda)(\underline{a}(\lambda)+\bar{a}(\lambda)) \mathrm{d} \lambda, \quad U_{L}(A)=\int_{0}^{1} L(\lambda)(\bar{a}(\lambda)-\underline{a}(\lambda)) \mathrm{d} \lambda
$$

Then $I_{L}(A)$ is called the centralized quantification principle value of $A$, and $U_{L}(A)$ is the dispersion of $A$. When $L^{*}=0$, we define $I_{L}(A)=[\underline{a}(1)+\bar{a}(1)] / 2$, and $U_{L}(A)=\bar{a}(1)-\underline{a}(1)$. Here, $A_{\lambda}=[\underline{a}(\lambda), \bar{a}(\lambda)]$ is the $\lambda$ - cuts of $A, L^{*}=\int_{0}^{1} L(\lambda) \mathrm{d} \lambda$.

Theorem 2 Let $A, B \in E^{1}, a, k \in \mathrm{R}$. Then:

1) $\left.\quad I_{L}(A \pm B)=I_{L}(A) \pm I_{L}(B), \quad U_{L}(A \pm B)=U_{L}(A)+U_{L}(B) ; 2\right) \quad I_{L}(k A)=k I_{L}(A), \quad U_{L}(k A)=|k| U_{L}(A)$ $I_{L}(a+A)=a+I_{L}(A) U_{L}(a+A)=U_{L}(A)$.

This theorem can be obtained by the definition of fuzzy number and the properties of integral.

Theorem 3 For $A=(a, b, c), \alpha \in[0, \infty)$, when $L(\lambda)=\lambda^{\alpha}$, we have

$$
I_{L}(A)=\frac{a+2 \alpha b+c}{2(\alpha+2)} \quad U_{L}(A)=\frac{c-a}{(\alpha+1)(\alpha+2)} .
$$

Theorem 3 can be proved by formula (1).

Generally speaking, in considering maximal (or minimum) fuzzy optimization problems, decision-makers always hope that $I_{L}(A)$ is as great (or small) as possible, the corresponding $U_{L}(A)$ is as small as possible simultaneously, but this case always can't be satisfied in practical problems. To establish a comparison method which can simultaneously consider $U_{L}(A)$ and $I_{L}(A)$, we introduce the concept of synthesizing effect function.

Definition2 A continuous function $S(x, y):(-\infty,+\infty) \times[0,+\infty) \rightarrow(-\infty,+\infty)$ is called a maximum synthesizing effect function, if it satisfies: 1) $S(x, y)$ is monotone non-decreasing on $x$ for any $y \geq 0 ; 2$ ) $S(x, y)$ is monotone non-increasing on $y$ for any $x \in(-\infty, \infty)$; 3$) S(x, y) \leq x, S(x, 0)=x$.

If we regard $x$ and $y$ as $I_{L}(A)$ and $U_{L}(A)$ of $A$ respectively, then 
$S_{L}(A)=S\left(I_{L}(A), U_{L}(A)\right)$

is a compound quantification method of fuzzy information considering both IL-metric and UL-dispersion. And this method not only contains IL-metric method, but it has better interpretability. In practical problems, we can choose different synthesizing effect function to embody different uncertainty conscious in the decision process.

\section{Location evaluate Approaches}

\section{Constructed Evaluation Index System}

The emergency logistics center is different from the common ones, and once it is built, it needs maintain and operating for a long period of time, and it must obey the four rules of being safe, being in time, having social benefits and being economical so as to provide effective rescue when emergencies happen. The factors that could influence the location of the logistics centers are complex with various values. It considers the technology, economy, environment and society, as indicated in "appendix table A". The AHP approach is taken to measure the hybrid multi-attribute index system. This essay takes the approach and conclusion of reference ${ }^{[15]}$, and the weight result is shown in "appendix table A".

Emergency Logistics Centers Evaluation Steps

Based on all above, a fuzzy multi-attribute decision-making approach with a unknown weight value is given. The steps as follows:

Step1 Switch the hybrid multi-attribute index value of the emergency logistics center with a triangular fuzzy number, and form a fuzzy decision-making matrix $A=\left(a_{i j}\right)_{m \times n}$ 。

Step2 the fuzzy decision-making matrix in the index layer $A=\left(a_{i j}\right)_{m \times n}$ may be changed into normalization matrix $\mathrm{B}=\left(\mathrm{b}_{\mathrm{ij}}\right)_{m \times n}$, among $\mathrm{b}_{\mathrm{ij}}=\left[\mathrm{b}_{i j}^{\llcorner}, \mathrm{b}_{i j}^{M}, \mathrm{~b}_{i j}^{\mathrm{U}}\right], \mathrm{b}_{i j}^{\llcorner}, \mathrm{b}_{i j}^{M}, \mathrm{~b}_{i j}^{\cup} \in(0,1], i=1,2, \ldots, m, j=1,2, \ldots, n$. The changing method is shown at $[14,16]$.

Step3 Clarify the triangular fuzzy numbers based on the combined quantity approach for the fuzzy numbers, that is, use formula(3)to calculate $I_{L}\left(B^{\circ} \rho, U_{L}\left(B^{\circ} \rho\right.\right.$, according to formula(4) the synthetic effect function $S\left(I_{L}\left(B^{\prime} \varphi, U_{L}(B / 9)\right.\right.$, it is shorted as $S(\cdot)$;

Step4 Use $f_{i j}=\sum_{j=1}^{n} S(\cdot) w_{j}$ to calculate the attribute value matrix ${ }_{\left[f_{i j}\right]_{m \times n}}$ in the norm layer.

Step5 Use $F=W_{1 m} *\left[f_{i j}\right]_{m \times n}$ to calculate the evaluation value of the projects and the project for the location of the emergency logistics centers could be got according to the ranking of the evaluation value.

\section{Case analysis}

The work to choose a location for the emergency logistics center in a region has started, and the experts suggest choosing one from the 6 projects available. According to the location- design rules as well as the index system for the emergency logistics centers, the qualitative index and the subjective entropy weight are calculated by the experts. The data in appendix table A is collected from China Statistical Yearbook in 2014.

In case, let $S(x, y)=x\left[1+a y\left(1+\kappa \cdot e^{\beta x}\right]^{b}\right.$ to be maximum effect function, which covers the dynamic relaxation features of $I_{L}(A)$ to $U_{L}(A), a, b$ reflects the punishment degree, $\kappa, \beta$ reflects the dynamic requirement for $I_{L}(A)$ to $U_{L}(A)$. To exam the above evaluation project, suppose $\theta=0.8, a=0.08$ $b=0.05, \kappa=0.5, \beta=0.8$, we have the following result through MATLAB.

Table1.The comprehensive evaluation results of the candidate emergency logistics centers

\begin{tabular}{|l|c|c|c|c|c|c|}
\hline Locations & $\mathbf{A 1}$ & $\mathbf{A 2}$ & $\mathbf{A 3}$ & $\mathbf{A 4}$ & A5 & A6 \\
\hline Comprehensive result & 0.0954 & 0.1123 & 0.1101 & 0.0594 & 0.0686 & 0.0784 \\
\hline Ranking & 3 & 1 & 2 & 6 & 5 & 4 \\
\hline
\end{tabular}

From the calculation results in Table 1, the ranking of the 6 candidate projects are A2, A3,A1, A6, A5, A4, among which A2 is the best. According to the practical analysis of the emergency logistics centers, project A2 are superior to others in environmental factors, social factors while are only inferior 
to A3 and A1 in technology and economic factors but still meets the basic rules for designing the location of the emergency logistics centers. Therefore, the location design model of the emergency logistics centers basically satisfies the reality.

\section{Conclusion}

When designing the location of the emergency logistics centers, we must obey the rules of being safe, being on time, paying attention to the social benefits as well as the economic factors. The decision-making ways for the location design of the emergency logistics centers reached the result anticipated through applying the synthetic effect information entropy and case analysis, therefore, they are scientific and practical. The location design for the emergency logistics centers shown in this essay overcomes the disadvantages of AHP weight and pays more attention to the experts' opinion; hence the decision is made more scientifically. It not only lays the foundation for the project choice and performance evaluation but also enriches the theory on information dealing and synthetic evaluation. It easily realizes the switch between the qualitative and quantity description, and blends effectively the decision-making consciousness into the decision-making process. Therefore, this essay is of great importance in solving the decision-making problems under uncertain circumstances.

\section{References}

[1] B.Balcik,B.M.Beatnon:Facility location in humanitarian relief.International Journal of Logistics:Research and Applicanons, Vol.11(2008),p.101-121

[2] T.T.Hu,P.Wan:A Brief Study on Emergency Logistics Centers Location Design based on Gravity Approach.Technological Development of Enterprise, Vol.31(2012),p.176-177

[3] T.Y.Zhou,P.Jian:A Study on the Establishment of a System of Location Decision Support for Emergency Shelter.Research of Soil and Water Conservation,Vol.8(2001),p.17-23

[4] X.Zhou,M.,Y.Wang:Study on the layout optimization theory of emergency shelter,"Journal of Safety and Environment,Vol.6(2006),p. 118-121

[5] O.Berman,Z.Drezner and Generalized coverage: new developments in covering location models:Computers\&Operations Research, Vol.37(2010),p.1675-1687

[6] R.Z.Farahani:Covering problems in facility location: a review. Computers\& Industrial Engineering, Vol.62(2012), p.368-407

[7] C.Zhang,S.Z.Chen, and P.H.J.Fu:In the Hierarchical Analysis of Emergency Materials Reserve Site Selection Study_Taking Zhejiang Province as an Example.Logistics Technology, (2014), p. 32-36

[8] L.Liang:Study on the Location of the Scheme Evaluation of Emergency Reserve Center.Logistics Engineering and Management, Vol.33(2011), p. 86-88

[9] L.Fang:Research on Location Model of Emergency System Preferences based on DEA.Systems Engineering Theory and Practice,Vol.8(2006), p. 116-122

[10] Y.F.Zhao,G.H.Chen :Emergency System Location Optimization Method based on the Improved TOPSIS (TOPSIS) Model.China Safety Science Journal,Vol.18(2008), p. 22-28

[11]Q.K.Cao,D.M.Song:Location of Emergency Logistics distribution center based on RS_AHP. Logistics Technology,Vol.32(2013), p. 103-105

[12]H.J. Zimmermann:Fuzzy set theory and its applications (Second Edition) .London: Kluwer Academic Publishers(1991).

[13]L.A. Zadeh:Fuzzy sets . Information and Control, Vol.8(1965), p. 338-353

[14]F.CLi,L.Li, C.X. Jin, R.J.Wang,and H.Wang:Research on the 3PL Supplier Selection Based on Set-Valued Satisfaction.Computers and Operations Research, Vol.39(2012), p. 1879-1884

[15] Y.F.Zhao,G.H.Chen:Location Model Design on Emergency System Optimization based on (TOPSIS) .China Safety Science Journal, Vol.18(2008), p. 22-28

[16]J.X.Wang, X.X.Zhu:Research on Emergency Logistics Center Location Decision Approach Based on Synthetic Information entropy,LISS(2015 ),P.351 
Appendix table A. Evaluation index Value for alternative Emergency Logistics Center

\begin{tabular}{|c|c|c|c|c|c|c|c|c|c|}
\hline \multicolumn{4}{|c|}{ Alternative Emergency Logistics Center } & $\mathrm{A} 1$ & $\mathrm{~A} 2$ & $\mathrm{~A} 3$ & A4 & A5 & A6 \\
\hline Criterion layer & Weigh & Index layer & Weigh & & & & & & \\
\hline \multirow{3}{*}{$\begin{array}{l}\text { Technology } \\
\text { Level }\end{array}$} & \multirow{3}{*}{0.5731} & The maximum passing time & 0.1645 & 20 & 22 & 24 & 28 & 22 & 20 \\
\hline & & Average passing time & 0.5386 & 18 & 16 & 15 & 15 & 17 & 18 \\
\hline & & Safety protection ability & 0.2969 & Medium & High & Low & Very High & Very Low & Medium \\
\hline \multirow{3}{*}{$\begin{array}{l}\text { Economic } \\
\text { Factors }\end{array}$} & \multirow{3}{*}{0.2532} & Fixed costs & 0.1667 & {$[835,855]$} & {$[1135,1144]$} & {$[1190,1210]$} & {$[1294,1307]$} & {$[998,1002]$} & {$[844,856]$} \\
\hline & & Emergency costs & 0.5000 & {$[370,385]$} & {$[320,330]$} & {$[314,322]$} & {$[300,310]$} & {$[315,320]$} & {$[360,380]$} \\
\hline & & Operating costs & 0.3333 & {$[255,265]$} & {$[190,210]$} & {$[175,185]$} & {$[215,224]$} & {$[220,240]$} & {$[235,255]$} \\
\hline \multirow{3}{*}{$\begin{array}{l}\text { Environmental } \\
\text { Factors }\end{array}$} & \multirow{3}{*}{0.1082} & Traffic conditions & 0.1571 & High & Medium & Very High & Medium & Low & High \\
\hline & & Features of the location & 0.4785 & Low & Medium & High & Very High & Medium & Low \\
\hline & & Coordinaton with the surroundings & 0.3644 & High & Low & Medium & Very Low & High & Medium \\
\hline \multirow{2}{*}{$\begin{array}{l}\text { Social } \\
\text { Factors }\end{array}$} & \multirow{2}{*}{0.0655} & Cultural level & 0.6667 & Very Low & Medium & High & Very High & Low & Medium \\
\hline & & Living conditions & 0.3333 & Medium & High & Medium & Very High & Medium & Low \\
\hline
\end{tabular}

